\title{
CÓMO PLANEAR UN PROGRAMA DE RADIO
}

Carlos Araya Rivera

\author{
Recibido 29-VII-2003 • Aceptado 12-VIII-2003
}

\begin{abstract}
Resumen: La planificación en la producción radiofónica es básica para lograr un mayor impacto de los mensajes. Este artículo explica una manera de diseñar un programa o espacio de radio, que pueda transmitirse en una estación cultural como las emisoras de la Universidad de Costa Rica, o una comercial. Se destaca la importancia de la investigación para fundamentar las propuestas, así como los elementos principales de un proyecto de programa radiofónico, que podrán formular profesionales y estudiantes de Comunicación o de cualquier otra disciplina del conocimiento.
\end{abstract}

Palabras clave: Comunicación, Producción Radiofónica, Planificación, Programa, Proyecto, Perfil.

\section{¿Planificar en radio?}

La producción de un espacio o programa radiofónico requiere de un proceso riguroso de planificación. La improvisación no tiene cabida, en tanto se hace necesario contar con una investigación que fundamente la propuesta del programa en términos de audiencia y de necesidades sociales de información. Además, se requieren planes de trabajo a corto y mediano plazo, estrategias de ejecución y espacios para la evaluación, entre otros aspectos.

Eduardo Contreras afirma que planificar es "pensar en un futuro, en algo que queremos lograr". Esto implica "ordenar una serie de pasos o etapas, tomar decisiones, asignar responsabilidades y fijar plazos para ejecutar actividades previstas" (1993, 58). Según Contreras, dichos pasos son el diagnóstico del problema, la definición de objetivos, el diseño de estrategias, la formulación del plan de trabajo, la implementación o búsqueda de los recursos, la ejecución y la evaluación. Por su parte, Arlette Pichardo (1994, 15-16) concibe la planificación como proceso y como sistema, ya que supone una secuencia lógica de etapas complementarias a la vez que establece una relación de espacios y plazos. Pichardo reduce las etapas del proceso a cinco: diagnóstico, formulación (de imagen-objetivo, estrategias, políticas y programación), ejecución, evaluación y ajuste. 
En el marco de la planificación de la comunicación, Carlos Sandoval (1990, 6) propone cuatro etapas: el diagnóstico, la programación, la ejecución y la evaluación. Con base en esta perspectiva, en un artículo anterior (Araya: 2000, 175-182) hablamos de la evaluación de los programas radiofónicos. En esta ocasión se explicarán el diagnóstico y la programación, esenciales para conocer con exactitud las características del público y así determinar el estilo y el ritmo del programa.

\section{Diagnóstico}

En el diagnóstico se definen las necesidades de información y comunicación que tiene determinado sector de población. Los datos se recopilan por medio de encuestas, entrevistas, grupos focales y análisis de mensajes. Daniel Prieto asegura que el diagnóstico es "una lectura esencial de determinada situación social, una lectura de sus conexiones esenciales, desde una perspectiva histórica” (1990, 41). Es decir, es un análisis detallado de una situación ubicada en un espacio y un tiempo específicos.

El proceso de diagnóstico puede iniciarse desde dos perspectivas (Araya y Montero: 1997, 27): una es empírica, partiendo de la realidad. Es decir, se percibe una necesidad y con base en ella se elabora un diagnóstico que confirme la observación. La otra perspectiva es científica: se parte de un estudio científico previo (puede ser de otra disciplina), se selecciona una o más necesidades sociales demostradas y se prepara un diagnóstico de comunicación que detalle las necesidades de información.

En la producción radiofónica, la realización de un diagnóstico es fundamental para:

1 conocer las características de un determinado sector de población.

1 seleccionar el género radiofónico adecuado.
1

diseñar el perfil del programa radiofónico.

1 escoger una radioemisora adecuada al perfil de la audiencia.

$1 \quad$ elegir el horario de transmisión más adecuado.

Como se indicó, la etapa de diagnóstico también comprende el conocimiento de la audiencia. A partir de aquí, se facilita el diseño de la estructura, la investigación de los temas y la redacción de los guiones, así como la decisión sobre elementos básicos (duración, horario y frecuencia del programa, entre otros). En la medida en que conoce más las necesidades y las características de su público, una productora o un productor radiofónico logra atraerlo con mayor eficacia y eficiencia.

Pero ¿cómo conocer mejor a la audiencia de nuestro futuro o actual programa radiofónico? En las Ciencias de la Comunicación, los publicistas son quienes conocen mejor a su público, pues lo logran por medio de la segmentación y así obtienen una descripción bastante precisa del mercado de sus consumidores. De esta forma, tanto William Arens (2000) como William Stanton, Michael Etzel y Bruce Walker (2001) proponen cuatro criterios de segmentación: geográfica, demográfica, psicográfica y conductual. La segmentación geográfica se refiere a la región, la cantidad de habitantes de la ciudad o localidad, condición urbana o rural y el clima del lugar donde reside el público potencial. En cambio, el criterio demográfico está relacionado con el ingreso económico, la edad, el género, la etapa de vida familiar (soltero, casado, sin hijos, etc.), la clase social, el nivel educativo, la ocupación y el origen étnico.

Con respecto a la segmentación psicográfica, Arens indica que agrupa a las personas por su estructura psíquica, es decir, por sus valores, actitudes, personalidad y estilo de vida (actividades, intereses, 
opiniones). Finalmente, la segmentación conductual está determinada por variables como el estatus del usuario, el nivel de uso del producto o servicio, la ocasión de compra y los beneficios buscados (2000, 151-155).

Sin embargo, el estudio de la audiencia radiofónica no se restringe únicamente al campo publicitario. Para obtener una descripción lo más precisa posible del público al que se va a dirigir el programa de radio, se propone la elaboración de un perfil en las siguientes dimensiones:

$1 \quad$ Perfil geográfico: región de residencia, cantidad de habitantes de la localidad, condición urbana o rural.

$1 \quad$ Perfil demográfico: ingreso económico, edad, género, etapa de vida familiar (soltero, casado, sin hijos, etc.), clase social, nivel educativo, ocupación y origen étnico.

$1 \quad$ Perfil psicográfico: valores, actitudes, personalidad y estilo de vida (actividades, intereses, opiniones).

El público se divide en público meta y público bienvenido. El primero es el grupo objetivo definido en las tres dimensiones anteriores, es la población a la que efectivamente se dirige el mensaje. El segundo también está definido en las tres dimensiones citadas y se conoce como público indirecto o secundario, ya que lo integran personas que podrían estar interesadas en escuchar el programa, sin necesariamente pertenecer al público meta. Por ejemplo, si se diseña una radio-revista para un público meta formado por personas adultas mayores, el público bienvenido podrían ser los familiares y los cuidadores de esas personas. Igual en el caso de una serie dramatizada para niños de 5 a 12 años, el público bienvenido podrían ser los padres y madres de esos niños, educadores de primaria, niños menores de 5 años o incluso mayores de 12 .

\section{Programación}

Cuando se demuestra científicamente una necesidad social de comunicación e información, puede iniciarse la etapa de programación. Aquí se elabora el proyecto de producción radiofónica, es decir, el documento que define y organiza el programa de radio. Este documento responde al qué, el cómo y el por qué de la producción.

En el proyecto de producción se establecen los objetivos y las metas que se pretende alcanzar, se escoge el género, se diseña el perfil del programa, se preparan planes de trabajo y el cronograma. Aquí también se enumeran los recursos humanos, materiales y económicos que requiere el proyecto para su ejecución. Así las cosas, el documento completo contiene cuatro secciones: investigación, perfil, presupuesto y planes de trabajo.

\section{Investigación}

La sección de investigación es tan solo una reseña del diagnóstico o de una recopilación bibliográfica efectuados con anterioridad. No se trata de incluir toda la investigación realizada, solo se fundamenta adecuadamente y en forma sintética el proceso de producción. Esta investigación debe realizarse tanto sobre el contenido como sobre el público meta.

\section{Perfil}

El perfil es un esbozo que define las características del programa radiofónico, tanto en forma como en contenido. La mejor comparación que encontramos es la cédula de identidad: el perfil es la cédula de identidad de un programa de radio, pues incluye una serie de elementos que permanecerán invariables durante cierto tiempo y que crean identificación y familiaridad con el público meta. Dichos elementos son: 
Género: Siempre con base en los resultados del diagnóstico se escoge el género ideal para el público meta: radio-revista, reportaje, microprograma, comentario, cuña radiofónica, mesa redonda, dramatización, etc. La selección depende de la intención u objetivo, del estilo y el ritmo de vida de la audiencia, así como de los recursos con los que se cuente para producir el espacio.

Si se desea llegar a un público que solo escucha radio para oír música, lo más conveniente es encauzar los esfuerzos a la producción de un espacio breve, como un microprograma o una cuña. De igual manera, esa decisión podría verse modificada o confirmada de acuerdo con el material informativo disponible y el tiempo de transmisión que brinde la radioemisora.

Tipo: Se refiere al tipo de género (periodístico, educativo, de entretenimiento, comercial).

Nombre: Todo programa de radio debe tener un nombre original, creativo y único. Es recomendable evitar los nombres trillados o cajoneros, obvios y evidentes. "De todo un poco", "Novedades" y "Juventud en acción" fueron nombres creativos en un principio, pero con el correr del tiempo muchas personas comenzaron a usarlos indiscriminadamente, al punto de que hoy pueden referir tanto a un programa periodístico como a uno de entretenimiento.

Objetivo: El perfil debe incluir el objetivo general de comunicación. Este objetivo expresa la intención de satisfacer la necesidad de información que experimenta el sector poblacional escogido, y que se deduce del problema descubierto. Dicha intención puede ser formar, informar o entretener, e incluso puede ser aún más específica como analizar, interpretar, recrear o criticar.

Para efectos prácticos, no es necesario incluir objetivos específicos en el perfil, pero es bueno definirlos porque son los aspectos concretos que se derivan del objetivo general. Si se desea, los objetivos específicos pueden ubicarse en la justificación.

En síntesis, un programa de radio podría tener como objetivo general brindar una educación básica a los niños trabajadores de la calle, mientras que algunos de sus objetivos específicos serían: fomentar valores esenciales, instruir a los niños con los conceptos básicos de las letras y las ciencias y advertir a los niños sobre los posibles peligros que pueden enfrentar diariamente (Araya y Montero: 1997, 29).

Público meta: Como se mencionó anteriormente, el público meta es el segmento de población al que se dirige el programa de radio y que efectivamente lo escuchará.

Público bienvenido: Es la población que podría estar interesada en escuchar el programa, pero al cual no se dirige el espacio.

Horario y frecuencia de transmisión: Día, hora y periodicidad de la transmisión. En este último punto hay que observar que los programas pueden ser diarios, interdiarios (de día por medio, ya sea lunes-miércoles-viernes o martes-juevessábados), semanales o quincenales. La periodicidad quincenal ya supone un problema de fidelidad de escucha, pues la recordación es bastante baja. Ni qué decir de los programas mensuales, aunque en esta última categoría entran las emisiones especiales (efemérides, acontecimientos y otros).

Emisora: Aquí se menciona la estación de radio que transmitiría el programa, con indicación de la frecuencia en AM o FM (*).

AM (Amplitud Modulada) es la banda de radiodifusión estándar, con un rango de frecuencias entre 535 y 1705 Kilohertz. La radio en $\mathrm{AM}$ es muy susceptible a ruido y a desvanecimientos periódicos o fading. FM (Frecuencia 
Duración: Se indica cuánto dura realmente el programa, para lo cual debe consultarse a la emisora sobre el tiempo efectivo del programa. Así, un programa de 30 minutos en realidad debería durar entre 25 y 27 minutos reales puesto que la emisora ocupa entre 3 y 5 minutos para sus compromisos comerciales y señales de identificación.

Justificación: En este apartado se justifican todos los elementos anteriores, con una breve referencia a la investigación realizada. Se trata entonces de justificar por qué el género, por qué el nombre del programa, por qué el objetivo, la emisora, la duración, el público meta y el horario de transmisión. Como se mencionó, aquí pueden ubicarse los objetivos específicos del programa.

Resumen: El perfil debe incluir también una reseña del programa, explicando en forma breve cómo será en general. Aquí puede mencionarse el estilo de locución, el ambiente y el uso de los demás recursos radiofónicos. También se recomienda citar en este punto la duración total del programa y, si es el caso, el tiempo destinado a comerciales.

En el apartado de resumen es bueno también describir las metas que se propone alcanzar con el programa, o sea, los logros cuantificables en cantidad y tiempo: cuántos programas se realizarán y durante cuántas semanas, meses o años. La cantidad de programas producidos en un periodo dependerá de las necesidades del público meta y de los recursos humanos, materiales y económicos con que se cuente como productor o productora. Siguiendo con el ejemplo del programa para los niños de la calle, el diagnóstico podría demostrar que lo más conveniente es un espacio diario para darle

Modulada) es una banda que emplea frecuencias en el rango de 88 a 108 Megahertz. Libre de estática e interferencias, la radio en FM se caracteriza por su alta calidad y fidelidad de transmisión (Figueroa: 1997; 94-100). un carácter continuo y permanente a la experiencia educativa. Desde luego, habría que contar con el presupuesto necesario para realizar una producción tan intensa.

Secciones: Un programa con estructura más o menos compleja debe seccionarse, con el propósito de tratar los temas en forma eficiente. Es necesario hacer una lista de las secciones que tendrá el programa, con sus respectivos nombres, una breve descripción, la duración y una mención general a la temática. En cada sección se menciona el género que se usará para tratar los temas.

Estructura: La estructura es el "esqueleto" del programa, porque aquí se ubican las secciones, sus respectivas duraciones (tiempo de sección) y el tiempo que se acumula conforme avanza el programa (tiempo acumulado).

Hay que hacer un ordenamiento adecuado de las secciones, pues conforme avanza la transmisión del programa, el oyente se va adaptando psicológicamente a él. Por esta razón, comenzar el programa con una sección lenta provocará un efecto distinto que si hay mucho dinamismo en los primeros minutos.

Temas tentativos: En algunos casos, la emisora solicitará una lista de temas posibles para el programa, para valorar si el espacio responde a las necesidades del medio. En este caso, conviene incluirlo al final del perfil, a manera de anexo. Sin embargo y como recomendación basada en la experiencia, es preferible anotar no más de seis o siete temas, por un lado para no agotar las posibilidades y por otro para evitar competencia desleal o uso no autorizado de las ideas.

A manera de ejemplo de todo lo anterior, se presenta el perfil completo de un programa radiofónico real: la radio-revista CONTRASTES, que desde 1990 producen los estudiantes de la Escuela de Ciencias de la Comunicación Colectiva de la Universidad de Costa Rica (vea Cuadro Nº1). 
Cuadro $\mathrm{N}^{\circ} 1$

\section{UNIVERSIDAD DE COSTA RICA \\ ESCUELA DE CIENCIAS DE LA COMUNICACIÓN / RADIO U PROYECTO CONTRASTES}

\section{PERFIL DE CONTRASTES}

\section{GÉNERO:}

TIPO:

NOMBRE:

OBJETIVO:

PÚBLICO META:

PÚBLICO BIENVENIDO:

TRANSMISIÓN:

FRECUENCIA:

DURACIÓN:

JUSTIFICACIÓN:

RESUMEN:

\section{Radio-revista.}

Educativa y de entretenimiento.

\section{CONTRASTES.}

Orientar, informar y recrear a los jóvenes, de tal forma que se facilite su inserción en la Universidad de Costa Rica.

Jóvenes de uno y otro sexo, con edades comprendidas entre los 18 y 24 años, estudiantes universitarios de cualquier estrato socioeconómico y que residan en zonas urbanas del Gran Área Metropolitana.

Jóvenes de uno y otro sexo, con edades comprendidas entre los 15 y 18 años, estudiantes del cuarto ciclo de secundaria de cualquier estrato socioeconómico y que residan en zonas urbanas del Gran Área Metropolitana.

Radio U (101.9 FM), miércoles, 3:00 pm.

Semanal.

25 minutos.

Cuando los jóvenes terminan sus estudios de secundaria, se encuentran con que deben tomar varias decisiones. Una de las más importantes, es la relacionada con seguir estudios superiores, proceso que se realiza muchas veces sin la debida orientación.

Por estas razones, se propone CONTRASTES, una radio-revista informativa, educativa y de entretenimiento que facilitará la inserción en la Universidad, y que al mismo tiempo informará y divertirá al público en mención.

La información será útil para los jóvenes de cualquier estrato socioeconómico que vivan en las zonas urbanas del Gran Área Metropolitana. Hay que hacer notar que se seleccionó el público indicado tomando en cuenta la cercanía que tiene dicho segmento de población con respecto a los productores de esta radio-revista, quienes son los estudiantes de Comunicación de la UCR.

El programa se transmite en la emisora estudiantil de la Universidad de Costa Rica, Radio U (101.9 FM), debido a que su público meta corresponde al de la radiorevista. Se propone transmitir los miércoles a las 3 de la tarde, horario de alta audiencia en el segmento de población, pues el público meta puede escuchar el programa con mayor detenimiento, con lo que se toma en cuenta los hábitos de este público.

La duración del programa es de 25 minutos, con el objetivo de que sea manejable en términos de producción y transmisión.

Dos presentadores, hombre y mujer, comentan e introducen los temas del programa. El tono utilizado es ágil y dinámico, con un tratamiento fresco y amistoso. Un 
tema central se plantea como hilo conductor, que estará compuesto por comentarios rápidos sobre el acontecer universitario, nacional e internacional, que interese a los estudiantes. El género incluye efectos de sonido y música.

\section{SECCIONES:}

Música compacta

Fórmula IC

Laberinto

Sin Censura

Pretileando

Planeta Música
Información y música sobre un grupo o un solista de rock-pop en español. Se incluye una pieza completa, al final de la sección (5’30”).

CHARLA sobre información vocacional (carreras universitarias). La sección incluye entrevistas a profesionales y estudiantes. Otros recursos utilizados son efectos de sonido, música y ambientes. Cada quince días se alternará con Laberinto (2'30").

CHARLA sobre los servicios que ofrece la Universidad de Costa Rica a sus estudiantes (becas, bibliotecas, actividades culturales y recreativas, etc.). La sección incluye entrevistas a profesionales y estudiantes. Otros recursos utilizados son efectos de sonido, música y ambientes. Cada quince días se alternará con Fórmula IC (2'30").

COMENTARIO sobre un tema de actualidad, de interés para los estudiantes universitarios (2'00").

CHARLA DRAMATIZADA sobre temas de interés personal: sexualidad y salud, uso del tiempo libre (deportes, pasatiempos, actividades culturales, etc.), comunicación y relaciones interpersonales, psicología práctica (estrés, autoayuda, etc.) (2’30”).

Información y música sobre un grupo o un solista de rock-pop en inglés u otro idioma. Se incluye una pieza completa, al final de la sección (5’30").

Estructura de CONTRASTES

\begin{tabular}{llr}
\hline Sección & T.S. & T.A. \\
\hline Saludo y resumen & $1: 00$ & $1: 00$ \\
Identificación & $0: 30$ & $1: 30$ \\
Sección de música & $5: 30$ & $7: 00$ \\
Hilo conductor-tema & $1: 00$ & 8.00 \\
Cortina & $0: 10$ & $8: 10$ \\
Fórmula IC / Laberinto & $2: 30$ & $10: 40$ \\
Cortina & $0: 10$ & $10: 50$ \\
Hilo conductor - tema & $1: 00$ & $11: 50$ \\
Cortina & $0: 10$ & $12: 00$ \\
Sin Censura & $2: 00$ & $14: 00$ \\
Mantenimiento & $0: 15$ & $14: 15$ \\
Hilo conductor - tema & $1: 00$ & $15: 15$ \\
Cortina & $0: 10$ & $15: 25$ \\
Pretileando & $2: 30$ & $17: 55$ \\
Cortina & $0: 10$ & $18: 05$ \\
Hilo conductor - tema & $1: 00$ & $19: 05$ \\
Cortina & $0: 10$ & $19: 15$ \\
Sección de música y & $5: 30$ & $24: 45$ \\
Despedida & & \\
\hline
\end{tabular}

T.S.: Tiempo de Sección

T.A.: Tiempo Acumulado

Fuente: Araya Rivera, Carlos. Inédito. 2002. 


\section{Presupuesto}

Para poder ejecutar lo planeado se necesita contar con recursos humanos, materiales y económicos. Por lo tanto, el proyecto de producción debe contemplar también una cotización de todos los gastos en que se incurrirá durante el proceso. El presupuesto se divide en dos partes: el presupuesto de producción y el presupuesto para la pauta.

En el presupuesto de producción, se tiene en primer lugar los recursos humanos: las personas que están dispuestas a realizar el programa radiofónico (director, productor, musicalizador, locutores, técnicos de grabación, guionistas, reporteros, asistentes, etc.). Cada una de estas personas debe ser remunerada de acuerdo con su aporte a la producción y dicha remuneración debe indicarse en el presupuesto respectivo.

Así las cosas, el director, el productor, el técnico de sonido, los reporteros y los asistentes cobran por horas, mientras que los guionistas, los locutores y el musicalizador cobran por producto (por guión, por texto narrado, por musicalización completa). Debido a la naturaleza de su labor, el director trabaja menos horas que el productor; por eso en la industria radiofónica se acostumbra pagarle al primero una tercera parte más que al segundo con el fin de compensar su dedicación.

En el presupuesto de producción debe considerarse un rubro para los materiales que se usarán (cintas de grabación, discos, papel, otros utensilios de oficina), el equipo (grabadoras, computadoras, impresoras) y las horas de estudio de grabación. En este punto se recomienda incluir dentro del presupuesto la formulación del proyecto de producción, ya que es un esfuerzo que debe ser remunerado adecuadamente.

Finalmente, la puesta al aire de un programa radiofónico requiere dinero suficiente para la compra del espacio de transmisión en la emisora. El presupuesto de pauta contempla el precio del espacio de transmisión, que debe indicarse de acuerdo con la periodicidad del programa (por día, semana, mes y periodo completo de exposición). Es bueno formular el presupuesto de pauta aparte, para detallar los elementos anteriores. El monto final para la pauta puede indicarse más adelante con el monto requerido para la producción.

\section{Planes de trabajo}

Los planes de trabajo son instrumentos para organizar las actividades por ejecutar en un periodo determinado. Las metas y los objetivos propuestos se alcanzan de manera estructurada, sistemática y constante, en periodos que pueden ir desde la jornada diaria hasta la anual. Al elaborar un plan de trabajo, se toma en cuenta el tiempo, los recursos disponibles (humanos, materiales y económicos) y los objetivos.

En primer lugar, puede estructurarse un plan de trabajo a largo plazo. Puede ser de seis meses, un año o más tiempo. Lo que interesa es establecer las líneas generales de la producción, la orientación y la política editorial. Una actividad de este plan es la constante revisión y confrontación de los contenidos y las formas de cada plan de corto plazo, con la orientación y el perfil definido para el largo plazo.

A corto plazo, es conveniente elaborar un plan de edición y un plan de producción. El plan de edición es la programación de los temas específicos que se transmitirán en un programa determinado, el enfoque que se le dará, los plazos de cierre de edición y producción, y la asignación de responsabilidades. El concepto de cierre de edición nos viene del periodismo, y significa la fecha y hora límites para entregar los materiales que se utilizarán en la redacción del guión final de un programa. Un ejemplo de plan de edición es el que utilizan los estudiantes de Comunicación para la radio-revista $\mathrm{CON}$ TRASTES (vea Cuadro $\mathrm{N}^{\circ} 2$ ). 
Cuadro $\mathrm{N}^{\circ} 2$

UNIVERSIDAD DE COSTA RICA

ESCUELA DE CIENCIAS DE LA COMUNICACIÓN / RADIO U PROYECTO CONTRASTES

Radio-revista CONTRASTES

Plan de Edición

\begin{tabular}{|l|l|l|}
\hline EDICIÓN N & $\begin{array}{l}\text { CIERRE DE } \\
\text { EDICIÓN: }\end{array}$ \\
\hline REVISTA N & $\begin{array}{l}\text { TRANSMISIÓN: } \\
\text { Miércoles_ de } \_ \text {GUION: }\end{array}$ \\
\hline DIRECCIÓN: & PRODUCCIÓN: & de 200 \\
\hline PRESENTADORES: & \\
\hline
\end{tabular}

\begin{tabular}{|c|c|c|}
\hline SECCIÓN & CONTENIDO & \\
\hline $\begin{array}{c}\text { TEMA CENTRAL } \\
\text { (hilo conductor) }\end{array}$ & & \\
\hline Sin censura & & \\
\hline $\begin{array}{c}\text { Fórmula IC / } \\
\text { Laberinto }\end{array}$ & & \\
\hline $\begin{array}{c}\text { Planeta Música } \\
\text { Música compacta }\end{array}$ & & \\
\hline Pretileando & & \\
\hline
\end{tabular}

Fuente: Araya Rivera, Carlos. Inédito. 2002.

Por otra parte, el plan o cronograma de producción es aquel en el que se contemplan las actividades por realizar, las fechas en que se espera llevarlas a cabo y las personas responsables de cada actividad. Aquí se puntualiza la investigación de los temas por tratar, la elaboración de los segmentos del programa, la redacción del guión definitivo, la grabación del programa, su transmisión y su evaluación correspondiente. El plan de producción es una herramienta muy útil para ver en perspectiva la distribución de los temas a lo largo del periodo.
A criterio de algunos productores, también es posible elaborar un plan a mediano plazo, en el cual se establecen los temas que se tratarán durante cada mes. Otros consideran que este nivel no es más que una ampliación del plan a corto plazo (Araya y Montero: 1997, 32).

\section{Punto de partida}

Una vez diseñado el proyecto de producción radiofónica, finaliza la etapa de 
programación. A partir de este momento, la productora o el productor radiofónico inicia la ejecución con la confianza de tener un objetivo claro. Desde luego, pueden haber imprevistos en el proceso que obliguen a modificar los planes, pero una planificación correcta facilitará la producción del programa radiofónico propuesto.

Valga la ocasión para hacer notar que los elementos anteriores no constituyen una receta que deba seguirse al pie de la letra. Cada persona interesada en la producción radiofónica valorará cuáles son los aspectos que le competen y que son cercanos a su realidad inmediata. Cualquiera que sea el camino que cada uno elija, se recomienda plantear el programa en forma sintética y a la vez lo más preciso posible.

\section{Referencias bibliográficas}

Araya Rivera, Carlos. "El ritmo como instrumento de análisis de programas radiofónicos". En: Revista Educación, Universidad de Costa Rica, Volumen $24, \mathrm{~N}^{\circ} 2,2000$, pp. 175-182.

Araya Rivera, Carlos y Montero Bolaños, Fernando. La producción de una radiorevista como espacio de práctica para los estudiantes de Comunicación Colectiva de la Universidad de Costa Rica. Tesis de grado. San José: Escuela de Ciencias de la Comunicación Colectiva, Universidad de Costa Rica. 1997.
Arens, William F. Publicidad. Sétima edición. México D.F.: Editorial McGrawHill. 2000.

Contreras B., Eduardo. Evaluación de Proyectos de Comunicación. Quito: CIESPAL. 1993.

Figueroa Bermúdez, Romeo. ¡Qué onda con la radio! México D.F.: Alhambra Mexicana. 1997.

Pichardo Muñiz, Arlette. Planificación y programación social. Bases para el diagnóstico y la formulación de programas y proyectos sociales. San José: Editorial de la Universidad de Costa Rica. 1994.

Prieto Castillo, Daniel. Diagnóstico de comunicación. Mensajes instituciones, comunidades. Quito: CIESPAL. 1990.

Sandoval García, Carlos. Planificar la comunicación: conceptos, herramientas, desafíos. San José: Universidad de Costa Rica, Instituto de Investigaciones Sociales. Serie Contribuciones, $\mathrm{N}^{\circ}$ 6. 1990.

Stanton, William J., Etzel, Michael J. y Walker, Bruce J. Fundamentos de Marketing. Undécima edición. México D.F: Editorial McGraw-Hill. 2001.

Carlos Araya Rivera Profesor Escuela de Ciencias de la Comunicación Colectiva Universidad de Costa Rica 